\title{
Allowable Mechanical Stress Applied to a Spider's Lifeline
}

\author{
Shigeyoshi OSAKI ${ }^{\dagger}$ \\ Department of Chemistry, Nara Medical University, Kashihara, Nara 634-8521, Japan
}

(Received October 18, 2006; Accepted December 16, 2006; Published February 2, 2007)

KEY WORDS Spider Silk / Mechanical Stress / Spider's Weight / Cross-Section Area / Elastic
Limit Stress /
[doi:10.1295/polymj.PJ2006135]

The mechanical properties of materials are one of the most important physical functions when stretching or compression. The mechanical breaking stress and elastic modulus of materials have been studied from the viewpoint of limiting mechanical characteristics. The mechanical breaking stress reflects the maximal limiting value at which materials break under very high stress while the elastic modulus reflects the rigidity of materials under very low stress; however, these values are not necessarily the actual stress applied to the materials.

It is interesting to estimate the mechanical stress actually applied to materials and also to know whether only part of the full limiting mechanical characteristics is used. Spiders' draglines are appropriate for estimating actual mechanical stress since the total weight of a spider is actually applied to the draglines when it falls. If the actual stress applied to spiders' draglines is determined, we will be able to evaluate the extent of the actual stress, providing important information for developing new materials.

Spider silks have been studied for mechanical stress, ${ }^{1-3}$ the elastic modulus, ${ }^{4-6}$ strain energy, ${ }^{7}$ and effects of UV rays. ${ }^{8-11}$ Mechanical breaking stress has been also explained at the molecular level., ${ }^{4}$ The author previously provided a basis for the maximal efficient safety of a spider's mechanical lifeline, ${ }^{13,14}$ and also a safety coefficient of the lifeline for not only mature but also juvenile Nephila clavata spiders. ${ }^{15}$ However, there have been no reports on the actual stress applied to the draglines when a spider falls. It is valuable to determine the magnitude of the actual stress from a spider's weight applied to the draglines and to evaluate to what extent the draglines are able to be effectively used up to the limiting stress.

This paper describes the actual stress applied to Nephila clavata spider draglines when a spider falls and compares it with the elastic limit stress as reliable limit stress and also with the mechanical breaking stress.

\section{EXPERIMENTAL}

Samples used here were draglines, which act as a mechanical lifeline for spiders to support their weight, secreted out of female Nephila clavata (Japanese golden web spider) when falling from a wooden bar in order to avoid the mechanical hysteresis of the draglines ascribed to the stress above the elastic limit stress (ES). Here, ES is defined as the stress at the elastic limit point which the stress-strain behavior changes from linear to nonlinear (see Figure 1). Very fine draglines secreted out of the spider's glands were carefully sampled to measure stress-strain curves and also for scanning electron microscopic (SEM) observation. ${ }^{6}$ After we ascertained whether the samples were suitable from SEM photos, we determined the exact cross-sectional area $S$ from the diameter of the draglines by SEM and then the ES and the breaking

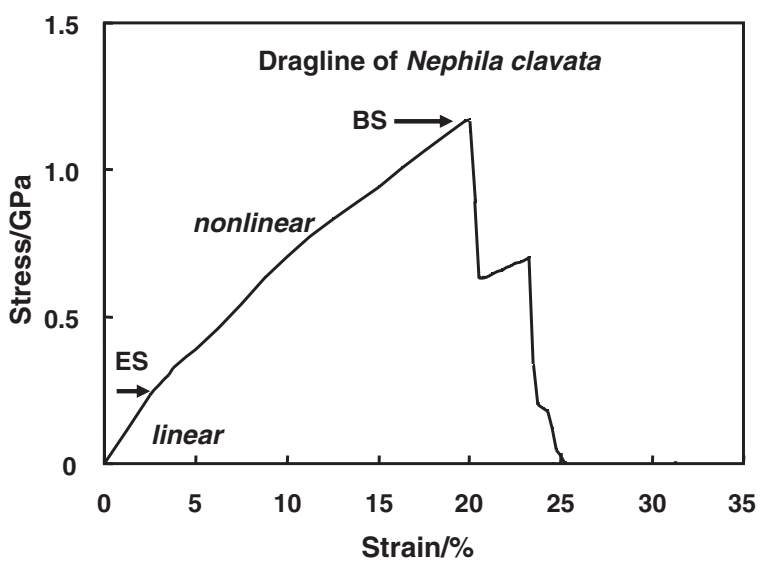

Figure 1. Stress-strain curve obtained for a dragline secreted from a Nephila clavata spider. Elastic limit stress (ES) and breaking stress (BS) are defined as the stress at the elastic limit point where the stress-strain behavior changes from linear to nonlinear and at the breaking point, respectively.

${ }^{\dagger}$ To whom correspondence should be addressed (Tel/Fax: +81-744-29-8810, E-mail: s-osaki@ naramed-u.ac.jp). 
stress (BS) from stress-strain curves of the draglines with a modified instrument (Instron, Japan), with a stretching velocity of $3.3 \times 10^{-4} \mathrm{~ms}^{-1}$. Here, BS is defined as the stress at the breaking point in the stress-strain curve (see Figure 1).

The draglines secreted from $N$. clavata spider are recognized as double filaments while they are usually observed as a single line with the naked eye. The filaments of draglines were ascertained to have almost a circular cross-section by SEM observation. ${ }^{14}$ Since cylindrical draglines have a minimum surface area, it is the most efficient structure for a spider to spin such lines.

The cross-sectional area $S$ of draglines was determined from the following equation:

$$
S=2 \Sigma s_{\mathrm{i}}=2\left\{\Sigma \pi\left(D_{\mathrm{i}} / 2\right)^{2}\right\} / n=\pi D^{2} / 2
$$

Here, $s_{\mathrm{i}}$ is the cross-sectional area of each filament, $D_{\mathrm{i}}$ is the diameter of each filament measured at different positions as the draglines, the subscript $\mathrm{i}(=1$ or 2$)$ represents each filament of double filaments and $n$ is the number of measurements. $D$ means diameter for double filaments. Here, $D_{\mathrm{i}}$ was measured at 6 points for each filament. The coefficient of variation in the observed diameter along the dragline was below 5\%.

The weight of $N$. clavata spiders in Japan increases exponentially from juveniles in spring to adults in autumn. ${ }^{6,14}$ For example, a spider's weight increases from several $\mathrm{mg}$ in spring, through about $50 \mathrm{mg}$ in August, up to about $1 \mathrm{~g}$ in late autumn. ${ }^{6}$

\section{RESULTS AND DISCUSSION}

Figure 2 shows the mean diameter $D$ of draglines plotted against the spider's weight $W . D$ increases not linearly but gradually with the increase in the spider's weight $W\left(1\right.$ gram $=9.8 \times 10^{-3}$ Newton $)$. This indicates that the $D$ of draglines increases with the growth of spiders but is not proportional to the $W$. Since the variation in the observed values of diameter

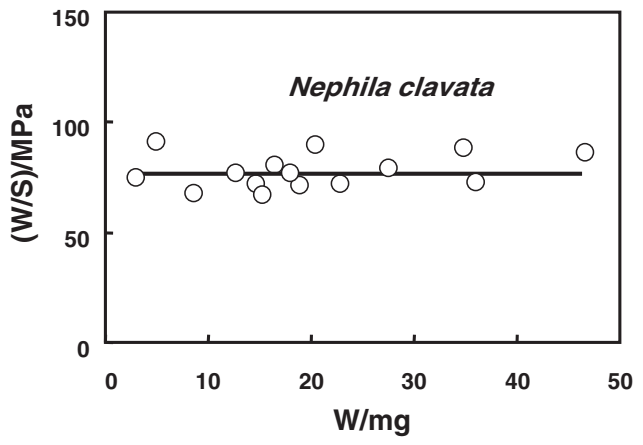

(a)

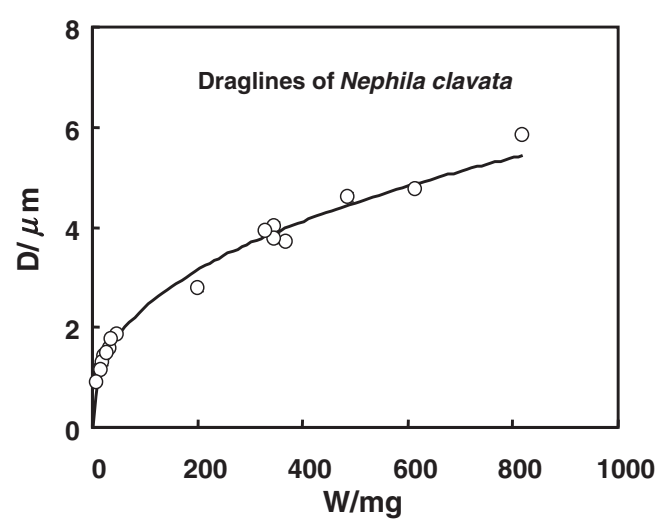

Figure 2. Mean diameter $D$ of draglines for Nephila clavata spiders plotted against spider's weight $W$.

was very low, the magnitude of variation was within circles drawn as data in Figure 1.

Next, we can observe a break at a weight of about $50 \mathrm{mg}$ in the graph of the cross-sectional area $S$ of draglines $v s$. the spider's weight $W . S$ increased linearly with increasing $W$. This indicates that the slope of $S$ vs. $W$ is almost constant, respectively, for juvenile spiders weighing less than $50 \mathrm{mg}$ and also for adult spiders weighing more than $50 \mathrm{mg} .{ }^{15}$ The value of about $50 \mathrm{mg}$ corresponds to the spider's weight in late summer when divergence of the sex into female and male occurs and then female's weight starts to increase rapidly. ${ }^{6}$

The total weight of a spider is applied to the dragline when falling. This means that the spider's weight can be supported only by the cross-section of draglines. Thus, it is valuable to determine the ratio of $W$ to $S$ of draglines.

Figures $3 \mathrm{a}$ and $3 \mathrm{~b}$ show $W / S$ plotted against the spider's weight $W . W / S$ was determined to be $c a$. $78 \pm 8 \mathrm{MPa}$ for juvenile spiders weighing less than $50 \mathrm{mg}$ and to be $c a .155 \pm 12 \mathrm{MPa}$ for adult spiders weighing more than $50 \mathrm{mg}$. The results indicate that the spider glands are well devised so that the cross-

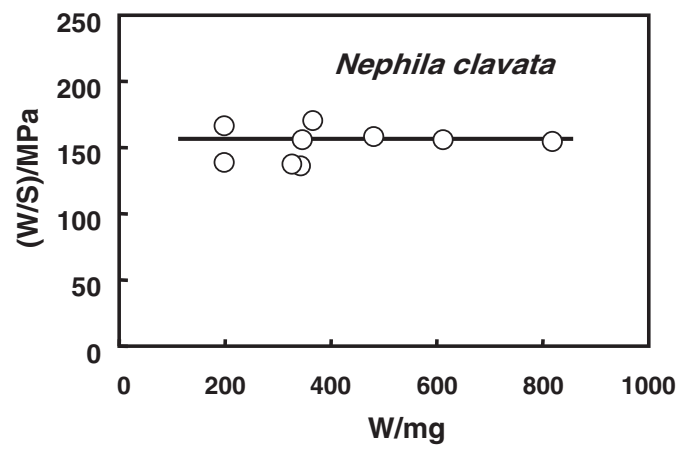

(b)

Figure 3. Spider's weight $W$ divided by cross-section area $S$ is plotted against spider's weight $W$. a, spiders weighing less than $50 \mathrm{mg}$. $\mathrm{b}$, spiders weighing more than $50 \mathrm{mg}$. 


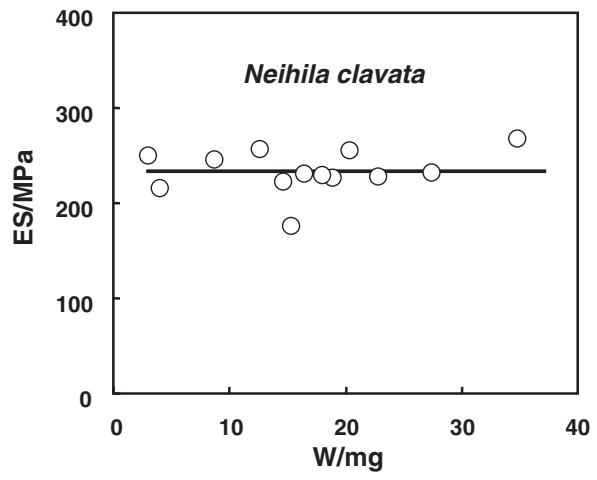

(a)

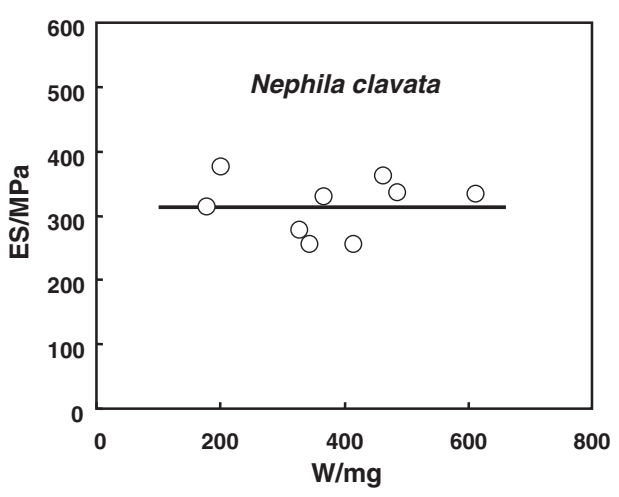

(b)

Figure 4. Elastic limit stress ES is plotted against spider's weight $W$. a, spiders weighing less than $50 \mathrm{mg}$. b, spiders weighing more than $50 \mathrm{mg}$.

sectional area of glands should increase linearly with increasing spider weight. And, the values of $W / S$ give meaningful data when evaluating the draglines as mechanical materials.

The stress-strain curve of draglines follows Hooke's law up to the elastic limit point at which the stress-strain behavior changes from linear to nonlinear. ${ }^{14}$ The elastic limit stress ES defined as the stress at the elastic limit point is considered to be reliable stress since stress-strain behavior follows Hooke's law up to the elastic limit point. That is, ES is considered as the maximal value at which spiders are able to trust draglines. Thus, it is significant to determine the values of ES as the reliable stress for $N$. clavata spider draglines.

Figures $4 \mathrm{a}$ and $4 \mathrm{~b}$ show ES plotted against a spider's weight $W$. ES was determined to be $c a .233 \pm$ $22 \mathrm{MPa}$ for juvenile spiders weighing less than $50 \mathrm{mg}$ and to be $c a .315 \pm 41 \mathrm{MPa}$ for adult spiders weighing more than $50 \mathrm{mg}$. The ES for juvenile spiders was smaller than that for adult spiders. This means that the draglines of juvenile spiders are safer than those of adult spiders since the value of $1 /(\mathrm{ES})$ reflects a measure of the safety of draglines.

If the stress applied to the draglines is smaller than the ES, spiders will be able to fall from draglines safely. Such $W / S$ values were smaller than those of the elastic limit stress ES. The stress due to the spider's weight applied to the draglines is about half of ES for adult spiders weighing more than $50 \mathrm{mg}$ and about $1 / 3$ of ES for juvenile spiders weighing less than $50 \mathrm{mg} .{ }^{15}$ The mechanical behavior of draglines when a spider falls is within the linear region of the stress-strain curve. Such results mean that spiders are able to trust draglines completely, and to move and fall safely by using their draglines.

On the other hand, the mechanical breaking stress (BS) and breaking strain of draglines as the limiting mechanical characteristics were determined to be
$1.25 \mathrm{GPa}$ (see Figure 2 in Ref. 10) and $c a .18 \%,{ }^{14}$ respectively. For comparison, the BS was several times larger than values of $600 \mathrm{MPa}$ for nylon and $400 \mathrm{MPa}$ for silkworm silk. The BS of spider draglines was much larger than the ES and $W / S$ values. Usually spiders will be not able to trust the BS as the whole of limiting mechanical characteristics of draglines safely because ultraviolet (UV) rays ascribed to sunlight change markedly the nonlinear behavior including the mechanical breaking stress in the stress-strain curve. ${ }^{15}$ For example, UV-A rays contribute to mechanically strengthen the dragline. The peak of BS increased rapidly after commencing irradiation, peaked fairly quickly, and then decreased gradually. ${ }^{15}$ On the other hand, the ES of draglines was never changed even though UV-A rays were irradiated to the draglines for a long time. ${ }^{16}$ The ES is able to be considered as the most reliable stress for spiders. Since the intensity of UV-irradiation from sunlight is dependent upon the weather, spiders could not predict when and where the nonlinear region in stress-strain curve would change and the draglines break. The fact that the nonlinear region in the stress-strain curve is large comparing with the linear one, may be required for corresponding to effects of environmental factors such as weather upon spider silk and also keep spider's life.

Thus, it was found that spiders usually use only part of the limiting mechanical characteristics from the values of ES and $W / S$ for spider draglines and their relationship. This means that a spider has the latitude to fall without using the total mechanical function of draglines.

This indicates that the limiting function for mechanical stress of spider draglines can be used in the event of danger which is related to environmental factors such as invaders, UV-irradiation, humidity and temperature. The extent of using mechanical function may be ascribed to the spider's long evolution of 400 million years. 
Finally, these results give useful information to understand to what extent mechanical function is used when a spider falls from a dragline, and important clues for evaluating the latitude of mechanical function required for developing new materials.

\section{REFERENCES}

1. M. J. Denny, J. Exp. Biol., 65, 483 (1976)

2. J. M. Gosline, M. W. Denny, and M. E. DeMond, Nature, 309, 551 (1984).

3. P. M. Cunniff, S. A. Fossey, M. A. Auerbach, and J. W. Song, in "Silk Polymers" D. Kaplan, W. W. Adams, B. Farmer, and C. Viney, Ed., ACS Symposium Series 544, Washington, 1994, p. 234.

4. Y. Termonia, Macromolecules, 27, 7378 (1994).
5. D. V. Mahoney, D. L. Vezie, R. K. Eby, W. W. Adams, and D. Kaplan, in "Silk Polymers" D. Kaplan, W. W. Adams, B. Farmer, and C. Viney, Ed., ACS Symposium Series 544, Washington, 1994, p. 196.

6. S. Osaki and R. Ishikawa, Polym. J., 34, 25 (2002).

7. A. Brandwood, J. Exp. Biol., 116, 141 (1985).

8. S. Osaki, Acta Arachnol., 43, 1 (1994).

9. S. Osaki, Acta Arachnol., 46, 1 (1997).

10. S. Osaki, Polym. J., 36, 657 (2004).

11. S. Osaki, K. Yamamoto, A. Kajiwara, and M. Murata, Polym. J., 36, 623 (2004).

12. A. H. Simmons, C. A. Michal, and L. W. Jelinski, Science, 271, 84 (1996).

13. S. Osaki, Nature, 384, 419 (1996).

14. S. Osaki, Int. Biol. Macromol., 24, 283 (1999).

15. S. Osaki, Polym. J., 35, 261 (2003).

16. S. Osaki, to be published. 